\title{
Parametrically shaped femtosecond pulses in the nonlinear regime obtained by reverse propagation in an optical fiber
}

\author{
Monika Pawłowska," Alexander Patas, Georg Achazi, and Albrecht Lindinger \\ Institut für Experimentalphysik, Freie Universität Berlin, Arnimallee 14, D-14195 Berlin, Germany \\ *Corresponding author: monika.pawlowska@fu-berlin.de
}

Received February 23, 2012; revised May 16, 2012; accepted May 17, 2012; posted May 18, 2012 (Doc. ID 163602); published June 26, 2012

\begin{abstract}
We present the experimental realization of a method to generate predetermined, arbitrary pulse shapes after transmission through an optical fiber in the nonlinear regime. The method is based on simulating the reverse propagation of the desired pulse shape in the fiber. First, linear and nonlinear parameters of a single-mode step-index fiber required for the simulation are determined. The calculated pulse shapes are then generated in a pulse shaper. @ 2012 Optical Society of America

OCIS codes: $190.4370,320.5540$.
\end{abstract}

While propagating in an optical fiber, femtosecond laser pulses with broad spectrum and high peak power are distorted by chromatic dispersion and nonlinear effects. Several methods for fiber delivery of ultrashort pulses have been proposed, including solitons [1], large mode area fibers [2], and combining the fiber with a dispersive element [3]. However, often it is desirable to have not just a transform limited pulse, but also to control the phase and amplitude. One possibility is an adaptive optimization method, but it requires a bulky pulse characterization setup after the fiber, which often defeats the purpose of using the fiber in the first place. In the linear regime, where only the dispersion has to be taken into account, it is enough to determine a phase offset once and then add it to any phase and amplitude pattern to obtain arbitrarily shaped pulses after transmission through the fiber [4]. If the shaped pulse propagates in a nonlinear medium, this approach is not valid.

In this Letter we demonstrate complex arbitrarily shaped pulses obtained after propagation through the fiber in the nonlinear regime using reverse propagation, a method proposed by Tsang et al. [5]. It relies on calculating the propagation of the desired pulse shape through the fiber backwards. The authors demonstrated the validity of this approach by characterizing the output pulse, simulating its reverse propagation, and then comparing the simulated pulse to the actual characterized input pulse. They proposed that a pulse shaper could be used to generate a calculated input pulse required to obtain a given output pulse. We discuss the practical aspects of this method: measurement of fiber parameters and adaptations of the algorithm that are necessary to apply it to pulse shaping and not just to numerically reproduce the measured pulse shapes. We present examples of parametrically shaped pulses that are relevant to coherent control: chirped single pulses as well as double pulses with variable distance.

The relative importance of linear and nonlinear effects for a pulse with peak power $P_{0}$ and duration $T_{0}$ can be estimated by calculating the ratio of the dispersion length and the nonlinear length, $L_{D} / L_{\mathrm{NL}}=\gamma P_{0} T_{0}^{2} /\left|\beta_{2}\right|$, where $\gamma$ is the nonlinear parameter related to the nonlinear refractive index $n_{2}$ and the effective mode area $A_{\text {eff }}$ by the relation $\gamma=\omega_{0} n_{2} / c A_{\text {eff }}$ and $\beta_{2}$ is the group velocity dispersion (GVD). For a fused silica fiber and a 50 fs Gaussian pulse, this ratio becomes close to 1 for pulse energy of the order of $0.1 \mathrm{~nJ}$.

Let us consider what happens to shaped pulses propagating in the fiber if only the dispersion is compensated for. This is demonstrated on two examples typical for coherent control experiments. In Fig. 1(a) pulses with varying linear chirp are shown. The self-phase modulation (SPM) combined with the negative chirp offset required to compensate for the positive material dispersion leads to spectral narrowing. The amount of narrowing depends on the linear chirp of the output pulse. In the second example, a pulse consisting of two subpulses with distance $\Delta t$ is generated by a periodical modulation of the spectral amplitude. As the spectral envelope experiences narrowing, the modulation pattern period decreases and as a result $\Delta t$ increases [see Fig. 1(b)]. It is clear that disregarding nonlinear effects and applying only linear dispersion compensation is not a good approach to obtaining arbitrarily shaped pulses after the fiber as SPM influences the pulse shape in a nonnegligible way.

The propagation of an ultrashort pulse in a nonlinear medium can be described by the nonlinear Schrödinger equation (NLSE) [1]

$$
\frac{\partial A}{\partial z}=\left[\frac{\alpha}{2}+\frac{i \beta_{2}}{2} \frac{\partial^{2}}{\partial t^{2}}-\frac{\beta_{3}}{6} \frac{\partial^{3}}{\partial t^{3}}+i \gamma|A|^{2}\right] A
$$
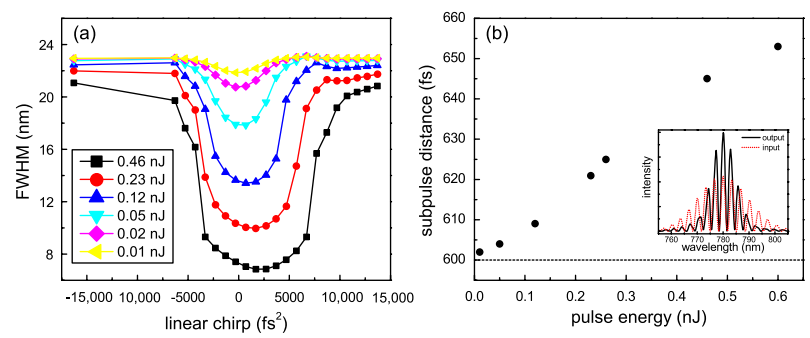

Fig. 1. (Color online) Influence of SPM on the pulse shape depending on pulse energy for (a) varying linear chirp and (b) two subpulses $600 \mathrm{fs}$ apart. 
where $A(z, t)$ is the slowly varying amplitude of the electric field, $\alpha$ corresponds to loss, and $\beta_{3}$ to third-order dispersion (TOD). The last term describes the SPM. $\gamma$ is assumed to be constant within the pulse bandwidth. In this Letter we consider only linearly polarized pulses propagating along one of the optical axes of the fiber. Self-steepening and intrapulse Raman scattering are negligible in the investigated pulse energy regime (a simulation of forward propagation shows less than $1 \%$ overall amplitude change).

The NLSE can be used to describe the propagation of a pulse in both directions. Backward propagation means reversing the sign of $z$, which is in turn mathematically equivalent to reversing the signs of the parameters $\alpha, \beta_{n}$, and $n_{2}$. Usually this means that the same code that is used for numerical forward propagation can be also used for reverse propagation simply by changing the signs of the input parameters that describe the fiber.

Contrary to adaptive optimization, which can produce desired pulses with no or very little knowledge of the fiber properties, reverse propagation requires precise knowledge of the parameters that appear in the NLSE. The values for bulk silica are not sufficient for a quantitative agreement with measurement. The chromatic dispersion of the fiber contains a waveguide contribution. As for $n_{2}$, many results obtained with different methods have been published [6], but results for femtosecond pulses at $800 \mathrm{~nm}$ are scarce. Moreover, it has been found that the nonlinearity of different samples of fused silica can differ by as much as 30\% [7]. In practice this means that the parameters must be determined for each fiber.

We measured the dispersion using a method first described in [8]. The spectral phase of a pulse entering the fiber is shaped so that the pulse length after propagation in the fiber is minimized. This is done by maximizing the signal from a nonlinear detector (a GaAsP photodiode). The pulse energy was reduced to suppress nonlinear effects. The optimal spectral phase obtained in this procedure is related to the dispersion of the fiber by the relation $\varphi^{\prime \prime}(\omega)=\beta^{\prime \prime}(\omega) L$, where $L$ is the fiber length. After verifying that adding further phase terms in the optimization does not further reduce the pulse duration, we express $\beta^{\prime \prime}(\omega)$ using the first two Taylor terms, GVD and TOD. The measured GVD $\left(46.7 \mathrm{fs}^{2} / \mathrm{mm}\right)$ is about $10 \%$ greater than the value for bulk fused silica $\left(37.8 \mathrm{fs}^{2} / \mathrm{mm}\right)$. This indicates that the waveguide contribution has the same sign as the material contribution, which is consistent with the measurement for a similar fiber included in [8]. Also the TOD value that we obtained $\left(137 \mathrm{fs}^{3} / \mathrm{mm}\right)$ is different from the one for bulk material $\left(27.0 \mathrm{fs}^{3} / \mathrm{mm}\right) . n_{2}$ was determined by simulating spectral narrowing for negatively chirped pulses. More precisely, we measured the spectrum after the fiber and then compared the spectrum obtained by reverse propagation with the actual input spectrum. The obtained value is $n_{2}=2.6 \times 10^{-20} \mathrm{~m}^{2} / \mathrm{W}$, which is consistent with previous measurements [?].

The parameters $\beta_{2}, \beta_{3}$, and $n_{2}$ are sufficient to calculate the input pulse shape that yields the desired output pulse. What is also required to determine the shaper transmission and phase mask is an exact knowledge of the input pulse spectrum, which can be influenced by coupling into the fiber. Apart from a constant loss that reduces the pulse energy, we observe spectrally dependent loss caused by space-time coupling, an inherent effect caused by coupling between the temporal phases of the pulse components and their wave vectors in the shaper setup [9]. In our case, the biggest contribution is the quadratic phase of around $-5 \times 10^{4} \mathrm{fs}^{2}$ that is needed to compensate the GVD of the fiber. It causes linear spatial chirp and thus limits the spectral bandwidth coupled into the fiber to about $20 \mathrm{~nm}$.

All simulations in this Letter were performed using LabVIEW. The split-step routine for simulating the propagation was taken from the Lab2 package [10]. The algorithm that converts a given pulse shape into transmission and phase patterns that can be applied on the pulse shaper was the same as, e.g., in [11], but there is more to consider. Reverse propagating a given output pulse results in an input pulse that has different spectral amplitude than the available laser pulse. This can be obtained by amplitude shaping, but then the pulse energy changes and the performed simulation is not valid anymore. To use exactly the right energy, one would have to know the spectral amplitude beforehand. One solution is repeating the simulation and reaching the correct pulse energy iteratively. We choose instead to assume a fixed loss due to amplitude shaping (or in other words a fixed overall shaper transmission) and use a modified pulse energy value for the simulation. The obtained transmission profile is then scaled down to fit the assumed total transmission. In cases where many different pulse shapes are calculated, such as genetic optimization, which is widely used in coherent control, the faster method is preferred.

Now we present some examples of shaped pulses obtained by reverse propagation. In each case, the pulse with the desired spectral phase and amplitude was numerically propagated backward through the fiber and the required shaper function was calculated as described above. For comparison, the pulses generated by neglecting the nonlinear effects and applying only the phase offset for chromatic dispersion compensation (referred to in the following as linear compensation) are also shown. The experimental setup, the same as in [12], consisted of a Ti:sapphire femtosecond oscillator delivering pulses at $780 \mathrm{~nm}$, a pulse shaper $(250 \mathrm{~mm}$ cylindrical lenses, and 1200 lines/mm gratings, and a liquid crystal spatial light modulator in $4 f$ configuration with $0.27 \mathrm{~nm} /$ pixel resolution) and a $1 \mathrm{~m}$ long single-mode step-index optical fiber (SM750, Fibercore Limited). The pulse energy after the fiber was limited to about $0.6 \mathrm{~nJ}$ because of the transmission of the shaper setup (mainly grating reflectivity) and the efficiency of coupling into the fiber. To reach higher pulse energies, some of the measurements were performed with amplified pulses at $792 \mathrm{~nm}$. In each case, a phase offset was determined to remove the residual chirp and obtain a transform limited pulse when no additional pattern was written on the modulator.

In Fig. 2 linearly chirped pulses with $10 \mathrm{~nm}$ spectral width, central wavelength of $780 \mathrm{~nm}$, and $0.47 \mathrm{~nJ}$ pulse energy are shown. For each pulse three cross-correlation traces, that is the ones for pulses generated with reverse propagation and with linear compensation only and the simulated trace of the desired pulse, as well as the corresponding spectra are shown. 

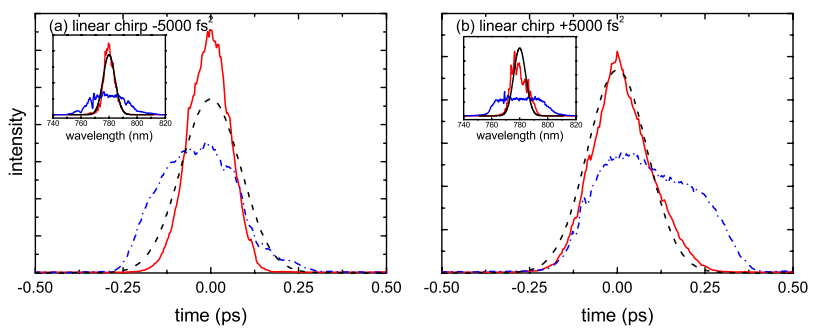

Fig. 2. (Color online) Measured cross-correlation traces of linearly chirped single pulses obtained by reverse propagation (red, solid) and by linear compensation (blue, dashed-dotted) compared to theoretical traces (black, dashed). Inset, corresponding spectra.

Next, some examples of double pulses are presented. Generating double pulses with a pulse shaper has several advantages, such as no need to look for the overlap as in a standard pump-probe experiment. Because of the loss due to amplitude shaping and of the distribution of energy between two temporally separated subpulses, one needs about four times more intensity to observe the same amount of nonlinear effects. To reach this intensity, we used amplified pulses attenuated so that in the absence of amplitude shaping, the total pulse energy was $2.2 \mathrm{~nJ}$. The spectral width of the subpulses after the fiber was again $10 \mathrm{~nm}$. Figure 3 shows the cross-correlation traces of pulses obtained by reverse propagation, by linear compensation, and simulated traces.

In all measurements presented here, the traces obtained by reverse propagation are much closer to the desired pulse shapes than those obtained by simple compensation of the fiber dispersion neglecting nonlinear effects. The possible reasons of the observed discrepancies are uncertainty in the measurements of fiber and pulse parameters and the finite resolution of the shaper. Nevertheless, the measurements presented here are a proof of principle that reverse propagation is suitable for obtaining arbitrarily shaped pulses after transmission through the fiber in the nonlinear regime.

In summary, we have shown experimentally that numerical reverse propagation combined with a pulse shaper, as proposed in [5], is a suitable method for generating parametrically shaped ultrashort pulses in a fiber where dispersion and SPM are present. We have presented examples of such pulses obtained with this method after propagation through a step-index optical fiber in the nonlinear regime. We demonstrated the capability to
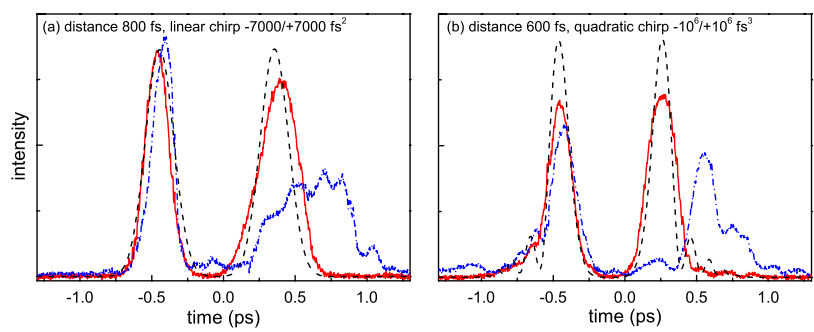

Fig. 3. (Color online) Measured cross-correlation traces obtained by reverse propagation (red, solid) and by linear compensation (blue, dashed-dotted) compared to theoretical traces (black, dashed) for double pulses with subpulses with (a) opposite linear chirp and (b) opposite quadratic chirp.

generate pulse shapes relevant for coherent control, such as linearly chirped pulses and pulses consisting of two subpulses with changeable distance in time. In all presented examples, the result obtained with reverse propagation were clearly better than those obtained by neglecting nonlinear effects. The proposed method provides new perspectives for endoscopic applications of ultrashort shaped laser pulses.

We thank Prof. Ludger Wöste for support. M. P. acknowledges the financial support of Studienstiftung des deutschen Volkes.

\section{References}

1. G. Agrawal, Nonlinear Fiber Optics (Academic, 2007).

2. T. Le, G. Tempea, Z. Cheng, M. Hofer, and A. Stingl, Opt. Express 17, 1240 (2009).

3. A. M. Larson and A. T. Yeh, Opt. Express 16, 14723 (2008).

4. F. Weise, M. Pawłowska, G. Achazi, and A. Lindinger, J. Opt. 13, 075301 (2011).

5. M. Tsang, D. Psaltis, and F. G. Omenetto, Opt. Lett. 28, 1873 (2003).

6. D. Milam, Appl. Opt. 37, 546 (1998).

7. S. Santran, L. Canioni, L. Sarger, T. Cardinal, and E. Fargin, J. Opt. Soc. Am. B 21, 2180 (2004).

8. G. Stobrawa, M. Hacker, R. Netz, M. Bischoff, and R. Sauerbrey, Appl. Phys. B 76, 333 (2003).

9. F. Frei, A. Galler, and T. Feurer, J. Chem. Phys. 130, 034302 (2009).

10. B. Schmidt, M. Hacker, G. Stobrawa, and T. Feurer, Lab2a virtual femtosecond laser lab, http://www.lab2.de/.

11. F. Weise, M. Pawłowska, G. Achazi, and A. Lindinger, J. Opt. Soc. Am. B 28, 406 (2011).

12. M. Pawłowska, A. Patas, G. Achazi, N. Rahmat, F. Weise, and A. Lindinger, J. Opt. Soc. Am. B 29, 833 (2012). 